\title{
Assessment of Diabetes Knowledge Using the Michigan Brief Diabetes Knowledge Test Among Patients With Type 2 Diabetes Mellitus
}

\author{
Turki M. Almalki ${ }^{\mathrm{a}}$, Naif R. Almalki ${ }^{\mathrm{a}}$, Khalid Balbaid ${ }^{\mathrm{b}}$, \\ Khaled Alswat ${ }^{\mathrm{a}, \mathrm{c}}$
}

\begin{abstract}
Background: Type 2 diabetes mellitus (T2DM) represents a growing health threat globally. The International Diabetes Federation (IDF) estimated that 387 million adults had diabetes in 2014, and this number is expected to continue to grow. In Saudi Arabia, the prevalence of T2DM over time has increased. Diabetes knowledge has been shown to improve self-management skills and glycemic control. The primary goal of this study was to assess diabetes knowledge and its impact on diabetes control and complications.
\end{abstract}

Methods: We conducted a cross-sectional study at King Abdulaziz Specialist Hospital, Taif City, Saudi Arabia, Division of Endocrinology. T2DM patients older than 18 years who underwent a routine visit to the endocrine clinic between June and October 2014 were asked to participate. Baseline characteristics and measurement were obtained at the time of visit. Laboratory data were collected from the patients' medical records. We excluded patients with type 1 diabetes mellitus (T1DM). We used the Michigan Brief Diabetes Knowledge Test to assess patients' knowledge. Those patients who answered $\geq$ $65 \%$ of the questions were considered to possess good knowledge about diabetes.

Results: A total of 264 patients participated; 131 (49.6\%) were male and $132(50.0 \%)$ were female. Approximately, half of the patients $(44.7 \%)$ had had diabetes for at least 10 years, and $29.8 \%$ of patients had had the disease for $5-10$ years. The cohort's mean A1c was $8.56 \%$ and mean body mass index was $30.5 \%$. Sixty-four percent of patients had only a high school education or less, and $38 \%$ had at least a college degree. Approximately, half of the cohort (41.7\%) were considered to be low income, $37.9 \%$ were on oral medications only, and $41.3 \%$ were on insulin. The mean fraction of correctly answered knowledge questions was $48.26 \%$. Twenty-eight percent of the participants thought that A1c reflected blood glucose control over the past week, and $44.3 \%$ did not know what A1c was. Approximately,

Manuscript submitted November 20, 2017, accepted December 5, 2017

aTaif University School of Medicine, Taif, Saudi Arabia

bKing Abdulaziz Specialist Hospital, Taif, Saudi Arabia

${ }^{c}$ Corresponding Author: Khaled A. Alswat, Taif University School of Medicine, Taif, Saudi Arabia. Email: kalswat@hotmail.com

doi: https://doi.org/10.14740/jem473w one-third of patients $(29.5 \%)$ believed that diet soda could be used to treat low blood glucose. Fifty-seven patients $(21.6 \%)$ were considered to have good knowledge about diabetes.

Conclusion: The majority (78.4\%) of the screened T2DM patients had poor knowledge about diabetes. Poor knowledge was associated with higher A1c, a non-significant increase in the majority of measured cardiovascular markers, and less awareness of diabetes-related complications.

Keywords: Diabetes knowledge; Type 2 diabetes; Awareness; Assessment

\section{Introduction}

Diabetes is a common disease worldwide. The International Diabetes Federation (IDF) has subsequently released estimates of the numbers of people living with diabetes in 2003 and a forecast of the affected population in 2025 (194 million and 334 million, respectively) [1]. Although the prevalence of diabetes mellitus (DM) is high among populations in the Middle East and Gulf countries, patients often lack the knowledge and skills to self-manage their condition [2]. Almost three decades ago, the population of the Kingdom of Saudi Arabia went huge changes in lifestyle, primarily leading to decreased physical activity and unhealthy eating habits [3]. These changes have had a considerable negative impact on the health of the society. Indeed, this lifestyle transformation is believed to be responsible for the epidemic of non-communicable diseases and their complications in the country [3]. It is thought that effective diabetes education and knowledge can improve self-management skills and glycemic control for patients with type 2 diabetes mellitus (T2DM) [4] and positively affect the health system. However, there is still a lack of studies that have assessed awareness and knowledge among diabetic patients in Saudi Arabic and more specifically in Taif City despite the high prevalence. Cross-sectional studies have suggested that the prevalence of T2DM in Saudi ranges from $10 \%$ to $30 \%[4]$.

The management of DM largely depends on patients themselves [5]. Therefore, patient education is always an important element of DM management [5]. Diabetes knowledge 


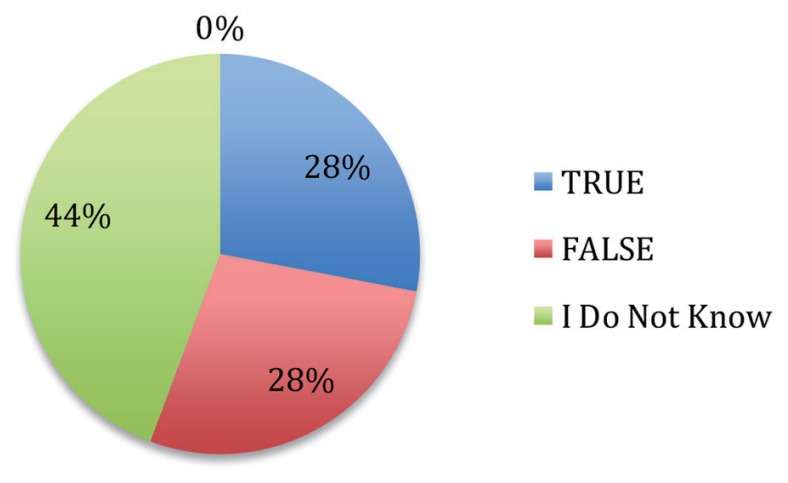

Figure 1. $\mathrm{HbA} 1 \mathrm{c}$ is a test that measures your average blood glucose level in the past week.

has been shown to improve self-management skills and glycemic control in patients with T2DM [4]. Several recent studies conducted in many parts of the world have suggested that there is a lack of public knowledge about various aspects of diabetes and that an enormous number of patients lack the skills to self-manage their condition [6]. The primary goal of this study was to assess diabetes knowledge and its impact on diabetes control and complications.

\section{Research Methodology}

A cross-sectional study was conducted between June 2014 and October 2014 at King Abdulaziz Specialized Hospital (KASH), Division of Endocrinology Taif City, Saudi Arabia. Random sampling was used to ensure that the participants were representative of the whole population. The study included 264 patients with T2DM older than 18 and who were willing to participate. We excluded patients with type 1 diabetes mellitus (T1DM) and patients with gestational diabetes. Data were collected through a formal interview with the patients conducted by one of the researchers. Baseline characteristics and measurements such as blood pressure, height and body weight were obtained at the time of visit. Laboratory data were collected from each patient's electronic medical record (EMR). This cross-sectional study used a questionnaire known as the Michigan Brief Diabetes Knowledge Test to assess each patient's knowledge [7-10].

This questionnaire was also translated into Arabic. The Arabic version of the questionnaire was used to reduce language barriers among patients. The questionnaire consisted of 20 true/false statements that aimed to assess DM knowledge and awareness. Patients who answered $>65 \%$ of the questions correctly were considered to possess good knowledge about DM. Socioeconomic data such as smoking, physical exercise, T2DM duration and education level were self-reported. All of the data were collected and analyzed using the statistical software SPSS version 20.0 (IBM, Chicago). We used frequency for category variables and mean and standard deviation (SD) for continuous variables. The Chi-squared test was used to study the relation between variables; we used the $t$-test to compare means. $\mathrm{P}$ value less than 0.05 is statistically sig-

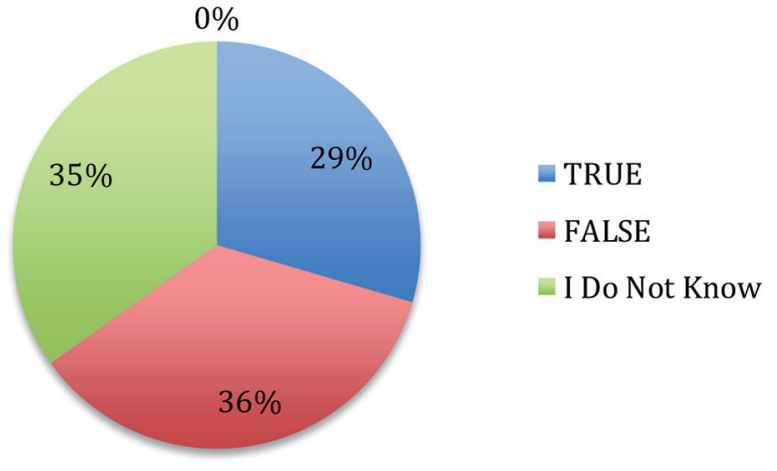

Figure 2. A can of diet soft drink can be used for treating low blood glucose levels.

nificant.

\section{Results}

A total of 264 patients participated, 131 (49.6\%) males and $132(50.0 \%)$ females. The mean age of the cohort was 53.2 years (SD: 13.4 years), and $44.7 \%$ of patients had had diabetes for at least 10 years. Approximately, one-third of patients $(29.8 \%)$ had had the disease for $5-10$ years. The mean A1c was $8.6 \%$, the mean body mass index (BMI) was $30.5 \%$, $66 \%$ of patients were married, $64 \%$ possessed a high school or lower level of education, $38 \%$ possessed at least a college degree, $41.7 \%$ were considered to be low income, $38 \%$ were on oral medications only, $41.4 \%$ were on insulin, $20.5 \%$ were on insulin \pm oral, and the mean fraction of correctly answered knowledge questions was $48.3 \%$. Approximately, one-third of the cohort (28\%) believed that A1c reflected blood glucose (BG) control over the past week and $44.3 \%$ of patients did not know what A1c was (Fig. 1). Approximately one in three patients believed that diet soda could be used to treat low BG, 34.8\% did not know and 35.6\% did not think so (Fig. 2).

Fifty-seven patients $(21.6 \%)$ were considered to possess good knowledge about diabetes. Compared with those with poor knowledge, diabetics with good knowledge of their disease had a mean age of 49 years vs. 54 years $(\mathrm{P}<0.05), 56.1 \%$ were male vs. $44 \%$ female $(\mathrm{P}=0.28)$, and $59.7 \%$ vs. $28.5 \%$ had a college degree or higher $(<0.05)$. Of diabetics, $76.4 \%$ with good knowledge reported a sedentary lifestyle compared with $85.5 \%$ who had poor knowledge $(\mathrm{P}=0.10)$. Approximately, $14.5 \%$ of the diabetics with good knowledge were active smokers compared with $13.5 \%$ of patients with poor knowledge $(\mathrm{P}=0.83)$. Diabetics with good knowledge had a mean A1c of 7.6 vs. $8.8(\mathrm{P}<0.05)$, a mean BMI of 30.6 vs. 30.5 , a mean systolic blood pressure (SBP) of 136.5 vs. $148.4(\mathrm{P}=$ $0.01)$, a mean resting heart rate of 73.1 vs. $73.8(\mathrm{P}=0.70)$, a mean total cholesterol (TC) of 167.2 vs. $176.3(\mathrm{P}<0.05)$, a mean LDL of 108.6 vs. 143.10 ( $\mathrm{P}<0.05)$, a mean HDL of 44 vs. $41(\mathrm{P}=0.20)$, and a mean triglycerides (TG) of $167.4 \mathrm{vs.}$ 183.6 $(\mathrm{P}=0.11)$. Table 1 and Table 2 show baseline characteristics of whole study. 
Table 1. Baseline Characteristics of the Entire Cohort

\begin{tabular}{|c|c|}
\hline Variables & \\
\hline Mean age in years $(\mathrm{SD})$ & \\
\hline Gender $(\%)$ & \\
\hline Male & $131(49.8 \%)$ \\
\hline Female & $132(50.2 \%)$ \\
\hline Duration of diabetes $(\%)$ & \\
\hline Fewer than 5 years & $67(25.6 \%)$ \\
\hline $5-10$ years & $78(29.8 \%)$ \\
\hline More than 10 years & $117(44.7 \%)$ \\
\hline Type of medication $(\%)$ & \\
\hline Oral hypoglycemic agents (OHAs) & $100(38 \%)$ \\
\hline Insulin & $109(41.4 \%)$ \\
\hline OHAs and insulin & $54(20.5 \%)$ \\
\hline Mean BMI (SD), kg/m² & $30.5(4.8)$ \\
\hline Mean $\mathrm{HbA1c}(\mathrm{SD}), \mathrm{mg} / \mathrm{dL}$ & $8.56(1.5)$ \\
\hline Mean systolic blood pressure (SD), $\mathrm{mm} \mathrm{Hg}$ & $141.5(17.6)$ \\
\hline Mean diastolic blood pressure (SD), $\mathrm{mm} \mathrm{Hg}$ & $78.14(9.2)$ \\
\hline Regular exercise $(\%)$ & $41(16.5 \%)$ \\
\hline Smoking $(\%)$ & $34(13.7 \%)$ \\
\hline Control of blood pressure & \\
\hline Controlled & $110(41.3 \%)$ \\
\hline Martial state (\%) & \\
\hline Single & $18(8.7 \%)$ \\
\hline Married & $174(84.1 \%)$ \\
\hline Divorced & $4(1.9 \%)$ \\
\hline Widow & $11(5.3 \%)$ \\
\hline Education level (\%) & \\
\hline Postgraduate & $9(3.4 \%)$ \\
\hline Graduated college & $84(32.1 \%)$ \\
\hline High school or less & $169(64.5 \%)$ \\
\hline Income & \\
\hline 5,000 SAR or below & $110(42.1)$ \\
\hline $5,000 \mathrm{SAR}-15,000 \mathrm{SAR}$ & $130(49.8)$ \\
\hline More than 15,000 SAR & $21(8)$ \\
\hline
\end{tabular}

All of the diabetics with good knowledge were aware that poorly controlled diabetes causes retinopathy compared with $74 \%$ of diabetes with poor knowledge $(\mathrm{P}<0.05)$ (Fig. 3$)$.

The corresponding data for coronary heart disease (CHD) and nephropathy were $82.5 \%$ vs. $55.2 \%(\mathrm{P}<0.05)$ and $87.7 \%$ vs. $75.7 \%(\mathrm{P}=0.13)$, respectively.

\section{Discussion}

The majority of studies have reported that diabetes knowl- edge and awareness is generally poor between T2DM patients [6-8]. However, it is difficult to compare our results with those of other studies that used different instruments to assess knowledge among diabetic patients. Our study revealed that the level of awareness and knowledge was low among diabetic patients. For example, $28 \%$ of the patients thought that A1c reflected BG control over the past week and $44.3 \%$ did not know what A1c was. Approximately, one-third of patients $(29.5 \%)$ thought that diet soda could be used to treat low BG, 34.8\% did not know and 35.6\% did not think so. We noted that most of the patients who were low income and had 
Table 2. Baseline Characteristics of the Entire Cohort

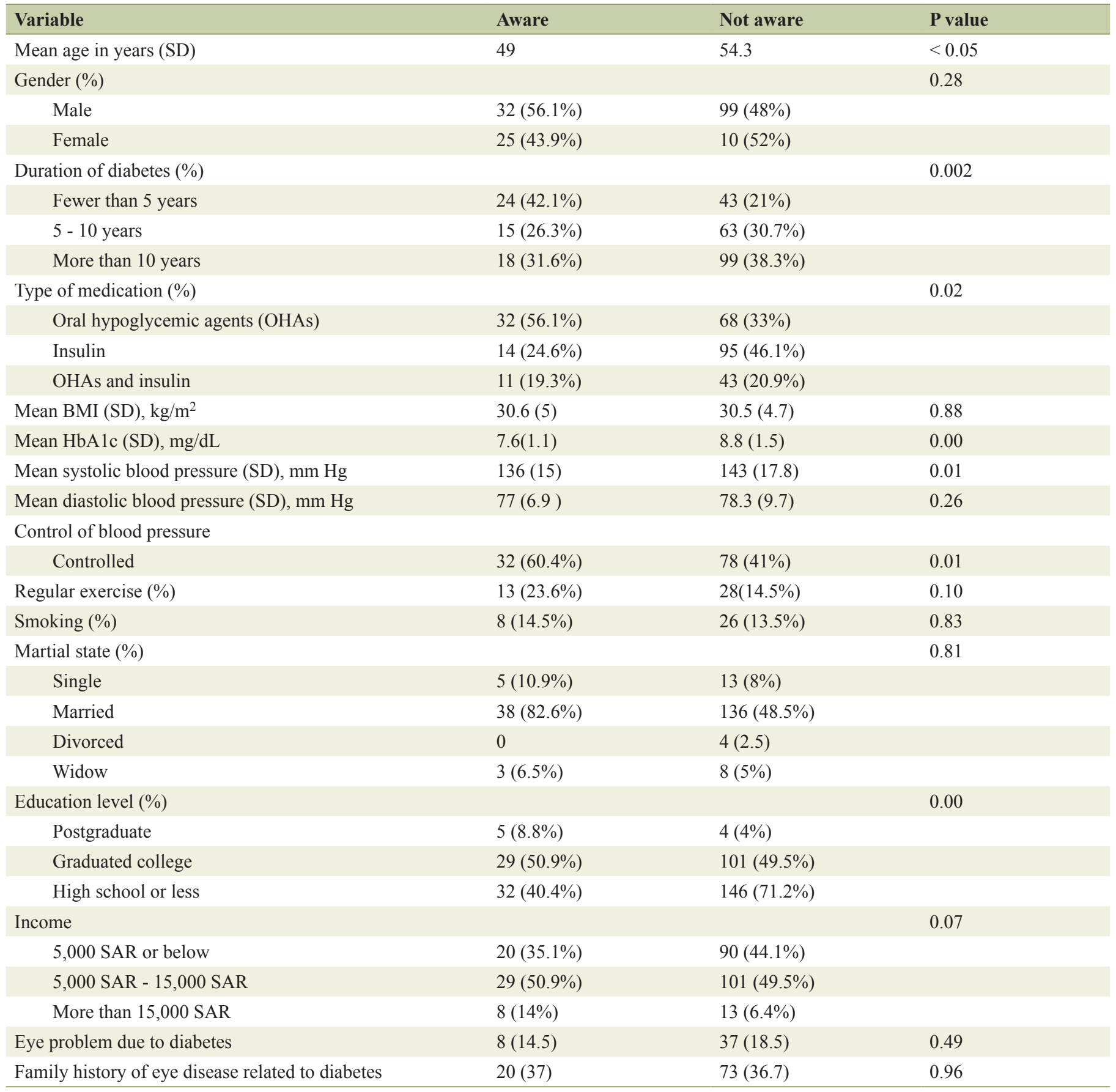

low education levels also had low awareness about diabetes complications. Therefore, these patients must be taught about their condition. Patients who have had T2DM for more than 10 years were less aware than those who have had T2DM for 5 - 10 years. Younger diabetic patients are more aware of their condition than elderly diabetic patients, but even patients who are aware of their diabetes report sedentary lifestyle, which gives us a clue that there is a gap between awareness and practice.
We found a correlation between high glycemic control and low level of knowledge among screened T2DM patients. However, some other studies have reported no correlation between these factors [9]. We believe that it is essential to direct more resources to improve knowledge because it has a very effective impact on improving patient compliance and selfcare. Since our study was outpatient hospital-based, we faced some limitations such as patients being unwilling to complete the questionnaire and incomplete laboratory findings in some 


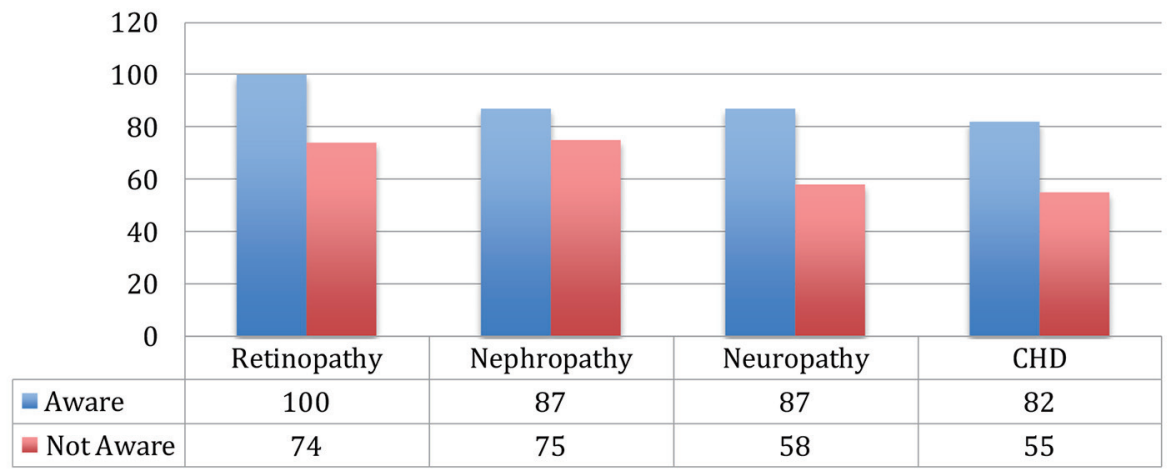

Figure 3. Complication awareness (\%).

patients' files.

\section{Conclusion}

The majority $(78.4 \%)$ of the screened T2DM patients were considered to have poor knowledge about diabetes. Poor knowledge was associated with higher A1c, a non-significant increase in the majority of measured cardiovascular markers, and less awareness about diabetes-related complications.

\section{Conflict of Interest}

None.

\section{Funding}

None.

\section{References}

1. Atlas D. Brussels. International Diabetes Federation. 2003.

2. Al-Adsani AM, Moussa MA, Al-Jasem LI, Abdella NA, Al-Hamad NM. The level and determinants of diabetes knowledge in Kuwaiti adults with type 2 diabetes. Diabetes Metab. 2009;35(2):121-128.
3. Alwan AD. Noncommunicable diseases: a major challenge to public health in the Region. Eastern Mediterranean health journal. 1997;3(1):6-16.

4. Norris SL, Engelgau MM, Narayan KM. Effectiveness of self-management training in type 2 diabetes: a systematic review of randomized controlled trials. Diabetes Care. 2001;24(3):561-587.

5. Powell CK, Hill EG, Clancy DE. The relationship between health literacy and diabetes knowledge and readiness to take health actions. Diabetes Educ. 2007;33(1):144-151.

6. Al-Khawaldeh OA, Al-Jaradeen N. Diabetes awareness and diabetes risk reduction behaviors among attendance of primary healthcare centers. Diabetes Metab Syndr. 2013;7(3):172-178.

7. The Michigan Diabetes Research and Training Center (MDRTC), University Michigan Health System website. Available: http://www.med.umich.edu/ mdrtc/profs/survey.html. (2013, accessed 4 Jun 2012).

8. Ben Abdelaziz A, Thabet H, Soltane I, Gaha K, Gaha R, Tlili H, Ghannem H. [Knowledge of patients with type 2 diabetes about their condition in Sousse, Tunisia]. East Mediterr Health J. 2007;13(3):505-514.

9. Khan LA, Khan SA. Level of knowledge and self-care in diabetics in a community hospital in Najran. Ann Saudi Med. 2000;20(3-4):300-301.

10. Al-Maskari F, El-Sadig M, Al-Kaabi JM, Afandi B, Nagelkerke N, Yeatts KB. Knowledge, attitude and practices of diabetic patients in the United Arab Emirates. PLoS One. 2013;8(1):e52857. 